\title{
Potentiation of cyclophosphamide chemotherapy using the anti-angiogenic drug thalidomide: Importance of optimal scheduling to exploit the 'normalization' window of the tumor vasculature
}

\author{
Jérome Segers $^{\mathrm{a}}$, Vincent Di Fazio ${ }^{\mathrm{b}}$, Réginald Ansiaux ${ }^{\mathrm{a}}$, Philippe Martinive ${ }^{\mathrm{c}}$, \\ Olivier Feron $^{\mathrm{c}}$, Pierre Wallemacq ${ }^{\mathrm{b}}$, Bernard Gallez ${ }^{\mathrm{a}, \mathrm{d}, *}$

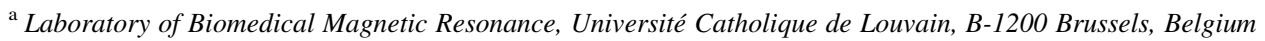 \\ ${ }^{\mathrm{b}}$ Laboratory of Drug Monitoring, Saint-Luc Hospital, Université Catholique de Louvain, B-1200 Brussels, Belgium \\ ${ }^{\mathrm{c}}$ Laboratory of Pharmacotherapy, Université Catholique de Louvain, B-1200 Brussels, Belgium \\ ${ }^{\mathrm{d}}$ Laboratory of Medicinal Chemistry and Radiopharmacy, Université Catholique de Louvain, B-1200 Brussels, Belgium
}

Received 22 September 2005; received in revised form 1 December 2005; accepted 5 December 2005

\begin{abstract}
The aim of this work was to study how administration schedule affects potentiation of cyclophosphamide, an alkylating agent, by thalidomide, an anti-angiogenic agent. Tumor oxygenation after thalidomide administration was determined over time by EPR oximetry. Such measurements provide a surrogate marker for determining the timing of 'normalization' of tumor vasculature. Re-growth delays were measured using different combinations and schedules of treatments. Additionally, the uptake of the metabolite of cyclophosphamide (hydroxycyclophosphamide or OH-CP) into tumors was determined by high performance liquid chromatography/tandem mass spectrometry (HPLC/MS/MS). A significant increase in $\mathrm{pO}_{2}$ was observed after 2 and 3 days of treatment before eventually declining on day 4. Thalidomide potentiated the effect of cyclophosphamide only when cyclophosphamide was administered after 2 days of treatment with thalidomide (no significant benefit using other schedules). In this time frame, the HPLC/MS/MS measurements showed that the quantity of OH-CP penetrating into the tumor was about twice in mice treated by thalidomide compared to controls. In conclusion, the present study demonstrates that the benefit of combined therapy using an anti-angiogenic agent with a cytotoxic agent requires knowledge of the time window during which the vessels initially become normalized.
\end{abstract}

(C) 2006 Elsevier Ireland Ltd. All rights reserved.

Keywords: Tumor microenvironment; Tumor oxygenation/perfusion; Chemotherapy; EPR; HPLC/MS/MS

\section{Introduction}

The ability of solid tumors to induce new blood vessel formation (angiogenesis) is crucial for tumor growth [1,2]. Anti-angiogenic therapies have been

\footnotetext{
* Corresponding author. Address: CMFA/REMA Units, Université Catholique de Louvain, Avenue E. Mounier 73.40, B-1200 Brussels, Belgium. Tel.: +32 2 7642792; fax: + 3227642790 .

E-mail address: gallez@cmfa.ucl.ac.be (B. Gallez).
}

proposed as cancer treatments, which gradually deprive a tumor of its blood supply. Despite promising results in experimental tumors $[3,4]$, the use of anti-angiogenic drugs alone has produced modest responses in clinical trials [5]. More recently, therapeutic strategies have evolved towards the combination of anti-angiogenic and cytotoxic therapies (radiation therapy or chemotherapy), which should provide the maximum benefit by targeting two separate compartments of the tumors: direct destruction of cancer cells by cytotoxic 
agents and indirect destruction by acting on endothelial cells [6]. However, this strategy may appear as counterintuitive: the destruction of the vasculature would impair the delivery of oxygen and chemotherapy to the solid tumor. This paradox may be resolved by the concept of 'normalization' of the tumor vasculature: the judicious application of the anti-angiogenic drug prunes the immature and inefficient blood vessels, leaving the more effective ones intact. This phenomenon should result in the enhancement of the delivery of drugs and oxygen into the tumor, thereby improving chemotherapy and radiation therapy $[6,7]$.

In a previous work [8], we showed that thalidomide radiosensitizes FSaII fibrosarcoma through early changes in the tumor microenvironment. The initial vascular 'normalization' by thalidomide accounted for the tumor radiosensitization because we demonstrated that thalidomide has no direct radiosensitizing effect, that tumor re-oxygenation occurs early after initiation of the treatment, and that these phenomena are correlated with an increase in perfusion and extensive vascular remodeling [8]. Here, we evaluated the effect of thalidomide on cyclophosphamide chemotherapy. For this purpose, we used another tumor model (hepatocarcinoma, TLT). This tumor model was selected because it was previously characterized for assessing the effect of treatments, which potentiate chemotherapy (P. Martinive, submitted). We first monitored the tumor $\mathrm{pO}_{2}$ over time after administration of thalidomide in order to measure the timing of the 'normalization' of the tumor vasculature in this tumor model. Using different combinations of treatments having different time frames of administration, we then measured tumor re-growth delays. Finally, we determined the uptake of the metabolite of cyclophosphamide (hydroxycyclophosphamide, or $\mathrm{OH}-\mathrm{CP}$ ) into tumors by high performance liquid chromatography/ tandem mass spectrometry (HPLC/MS/MS).

\section{Materials and methods}

\subsection{Mice and tumors}

Syngeneic transplantable mouse liver tumor (TLT, [9]) was injected intramuscularly in the thigh of 5-week-old male NMRI mice (Animalerie facultaire, Université catholique de Louvain, Brussels). For tumor oxygenation experiments, tumor bearing mice were anesthetized using isoflurane (Forène, Abott, Louvain-La-Neuve, Belgium) delivered using a calibrated vaporizer at $2.5 \%$ in air for induction and $1 \%$ for maintenance. For blood analysis, tumor bearing mice were anesthetized using I.P. injection of ketamine/xylazine $(80 \mathrm{mg} / \mathrm{kg}$ ketamine
[Imalgene, Lyon, France], $8 \mathrm{mg} / \mathrm{kg}$ xylazine [Bayer, Brussels, Belgium]). All animal experiments were performed in accordance with national animal care regulations.

\subsection{Tumor oxygenation}

Electronic paramagnetic resonance (EPR) oximetry, using charcoal (CX0670-1, EM Science, Gibbstown, NJ) as the oxygen sensitive probe, was used to evaluate the tumor oxygenation [10]. EPR spectra were recorded using an EPR spectrometer (Magnettech, Berlin, Germany) with a low frequency microwave bridge operating at $1.2 \mathrm{GHz}$ and extended loop resonator. Mice were injected once in the center of the tumor 1 day before measurement using the suspension of charcoal (suspension in saline containing 3\% Arabic gum, $100 \mathrm{mg} / \mathrm{ml}, 50 \mu \mathrm{l}$ injected, $1-25 \mu \mathrm{m}$ particle size). The localized EPR measurements correspond to an average of $\mathrm{pO}_{2}$ values in a volume of $\sim 10 \mathrm{~mm}^{3}$ [10]. Data acquisition was performed on a daily basis before administration of thalidomide in order to ensure that there were no acute effects of the treatment.

\subsection{Schedule of treatments}

Racemic thalidomide (Sigma-aldrich, Bornem, Belgium) was dissolved in DMSO and given via I.P. injection $\left(200 \mathrm{mg} / \mathrm{kg}\right.$ ). Cyclophosphamide (Endoxan ${ }^{\circledR}$, Baxter, Brussels, Belgium) was dissolved in saline and administered via I.P. injection $(50 \mathrm{mg} / \mathrm{kg})$. Re-growth delay experiments in TLT showed that this dose of $50 \mathrm{mg} / \mathrm{kg}$ is just below the efficacy threshold for this product (experiments performed with doses ranging from 10 to $250 \mathrm{mg} / \mathrm{kg}$ ). Treatments were started when tumors reached $8.0 \pm 1.0 \mathrm{~mm}$ and were performed as shown in Table 1. Tumors were measured daily with an electronic caliper until they reached a diameter of $15 \mathrm{~mm}$, at which time the mice were sacrificed.

\subsection{Extraction of hydroxycyclophosphamide from tumor and blood samples}

The protocol was adapted from Refs. [11-14]. Briefly, 30 min after injection of cyclophosphamide, mice were sacrificed and tumors carefully excised. The tumors were suspended in $5 \mathrm{ml}$ water and homogenized with Ultraturax. Ifosphamide (LGC Promochem, Molsheim, France), an analog of cyclophosphamide, was used as internal reference and spiked to a final concentration of $50 \mathrm{ng} / \mathrm{ml}$. To eliminate cellular debris, the suspension was centrifuged at $4{ }^{\circ} \mathrm{C}$ for $5 \mathrm{~min}$ at $2700 \mathrm{~g}$, and the supernatant was recovered. Acetonitrile (Lan Scan, Dublin, Ireland) was then added. The volume of acetonitrile added corresponded to three times the volume of the recovered supernatant. The mixture was then mixed using a vortex, and finally centrifuged at $4{ }^{\circ} \mathrm{C}$ for $5 \mathrm{~min}$ at $2700 \mathrm{~g}$. Eight milliliter of clear supernatant were recovered and then evaporated with a Speed Vaq (Savant, Farmingdale, NY). For HPLC/MS/MS analysis, the dry 
Table 1

Schedule of treatments

\begin{tabular}{llllll}
\hline & Day 1 & Day 2 & Day 3 & Day 4 & Day 5 \\
\hline Group 1 & DMSO & DMSO & & \\
Group 2 & Thalidomide & Thalidomide & & \\
Group 3 & DMSO & DMSO & Cyclophosphamide & \\
Group 4 & Thalidomide + & Thalidomide & & \\
& cyclophosphamide & & & \\
Group 5 & Cyclophosphamide & Thalidomide & Thalidomide & & \\
Group 6 & Thalidomide & Thalidomide & Cyclophosphamide & Thalidomide & Cyclophosphamide \\
Group 7 & Thalidomide & Thalidomide & Thalidomide & Thald
\end{tabular}

residues were finally reconstituted with $100 \mu \mathrm{l}$ water and $100 \mu \mathrm{l}$ acetonitrile. For blood analysis, a second series from the same batch of tumor bearing mice was used. The protocol was the same except that we used $500 \mu$ of blood samples (obtained by cardiac puncture), a centrifugation at $8500 \mathrm{~g}$, and $500 \mu \mathrm{l}$ of clear supernatant before evaporation using a Speed Vaq. For HPLC/MS/MS analysis, the dry residues were finally reconstituted with $100 \mu \mathrm{l}$ water and $100 \mu$ l acetonitrile.

\subsection{Liquid chromatography (LC)}

The chromatograph system was a Waters Alliance 2795 Separation Module (Milford, MA, USA) consisting of an XTerra $^{\circledR}$ MS C18 column (Waters) of $2.1 \times 50 \mathrm{~mm}$ and $3.5 \mu \mathrm{m}$ particle size with appropriated guard columns. The mobile phase was a constant mixture of $20 \%$ acetonitrile and $80 \%$ ammonium acetate buffer $(2 \mathrm{mM}, \mathrm{pH} 3)$ with $0.1 \%$ formic acid. A flow rate of $300 \mu \mathrm{l} / \mathrm{min}$ was able to eluate all target compounds during a run of $5 \mathrm{~min}$. The column was used at $50{ }^{\circ} \mathrm{C}$. The injection volume into the LC system was $20 \mu \mathrm{l}$ and pressure $\sim 1350$ psi.

\subsection{Mass spectrometry (MS)}

Detection was performed with a Waters Micromass Quattro micro spectrometer. The instrument operated in electrospray positive ionization mode and was directly coupled to the LC system. System control and data acquisition were carried out by MassLynx 3.5 software. The cone voltage and collision energy were optimized for each compound (see Table 2). Source and desolvatation temperatures were, respectively, 140 and $300{ }^{\circ} \mathrm{C}$. The electrospray capillary voltage was fixed at $3.5 \mathrm{kV}$. High purity nitrogen gas was used as nebulizer gas with a flow of $650 \mathrm{l} / \mathrm{h}$ and as cone gas with a flow of $50 \mathrm{l} / \mathrm{h}$. Collision gas pressure was about $2.5 \times$ $10^{-3}$ mbar argon. Sample analysis was performed in MRM (multiple reaction mode) with a dwell time of $0.5 \mathrm{~s}$ per channel. MS and MS/MS spectra were collected.

\subsection{Statistical analysis}

Results are given as mean \pm SEM values from $n$ animals. Comparisons between groups were made with Student's two- tailed $t$-test and a $P$ value less than 0.05 was considered significant.

\section{Results}

\subsection{Thalidomide treatment induced an early increase in tumor $\mathrm{pO}_{2}$ in TLT tumors}

EPR oximetry relies on the oxygen-dependent broadening of the EPR line width of a paramagnetic oxygen sensor implanted in the tumor [15]. This technique is designed for continuous measurement of the local $\mathrm{pO}_{2}$ without altering the local oxygen concentration, and allows repeated measurements from the same site over long periods of time. Daily thalidomide treatment modified the tumor $\mathrm{pO}_{2}$ in TLT tumors as shown in Fig. 1. The tumor $\mathrm{pO}_{2}$ before the treatment with thalidomide was $1.7 \pm 0.3 \mathrm{mmHg}(n=5)$. A significant increase $(P<0.05)$ in $\mathrm{pO}_{2}$ was observed after 2 days of treatment $(15.9 \pm 3.1 \mathrm{mmHg}) . \mathrm{pO}_{2}$ values remained elevated through day 3 , before eventually declining on day $4(1.50 \pm 0.17 \mathrm{mmHg})$. These results are comparable to our previous study using FSaII fibrosarcomas [8] and strongly suggest that the 'normalization' of the tumor vasculature after thalidomide treatment also occurs in the TLT tumor model.

\subsection{The efficacy of the combination thalidomidel cyclophosphamide was dependent on the schedule of administration}

We used the results from the evolution of tumor $\mathrm{pO}_{2}$ as the rational basis for defining different schedules of

Table 2

MRM parameters for the analytes during HPLC/MS/MS

\begin{tabular}{lllll}
\hline Analyte & $\begin{array}{l}\text { Precursor } \\
\text { ion }(\mathrm{m} / \mathrm{z})\end{array}$ & $\begin{array}{l}\text { Product ion } \\
(\mathrm{m} / \mathrm{z})\end{array}$ & $\begin{array}{l}\text { Collision } \\
\text { energy }(\mathrm{V})\end{array}$ & $\begin{array}{l}\text { Cone } \\
\text { voltage }(\mathrm{V})\end{array}$ \\
\hline $\mathrm{IF}$ & 261 & 91.8 & 30 & 22 \\
OH-CP & 277.0 & 142.0 & 25 & 45 \\
\hline
\end{tabular}

IF, ifosfamide; OH-CP, hydroxy metabolite of cyclophosphamide. 


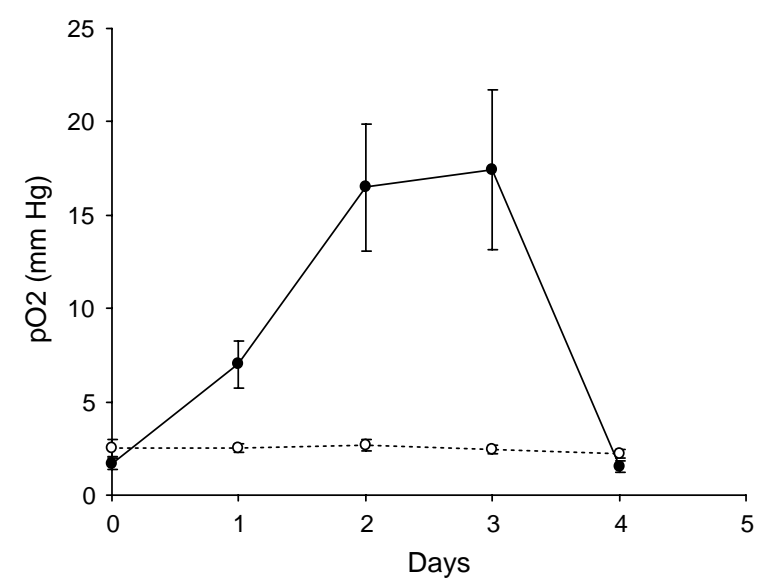

Fig. 1. Evolution of $\mathrm{pO}_{2}$ in tumors treated with daily injection of thalidomide (black) and DMSO (white).

treatments (see Table 1). Besides the control (group 1, $n=7$ ), thalidomide-alone (group $2, n=7$ ), and cyclophosphamide-alone (group 3, $n=7$ ) groups, we used four different combinations: cyclophosphamide with (group 4, $n=7$ ) or before (group 5, $n=8$ ) thalidomide treatment, and cyclophosphamide 2 days (group 6, $n=5$ ) or 4 days (group 7, n=8) after thalidomide treatment. The tumor size was monitored every day for all mice and the time for each tumor to reach $15 \mathrm{~mm}$ was calculated. For control group 1, this time was $8.59 \pm 0.82$ days. Fig. 2 shows the difference in this time between the control group and each of the six treatment groups (re-growth delay). These results show that thalidomide potentiated the effect of cyclophosphamide. The effect was not significant for groups 4,5 , and 7 . The potentiation was maximal for group 6 (when cyclophosphamide was administered after 2 days of treatment with thalidomide). The regrowth delay was significantly higher for group 6 than for all other groups $(P<0.05)$.

\subsection{The potentiation of the combination thalidomidel cyclophosphamide was explained by an increased delivery of the active metabolite of cyclophosphamide in tumors}

Because the potentiation effect of cyclophosphamide was maximal in mice treated with two daily injection of thalidomide (group 6), the amount of cyclophosphamide metabolite (OH-CP) that enters the cells was determined in this group of tumors (and in the corresponding control group, group 3). Fig. 3 shows a representative chromatogram of a tumor sample. The cone voltage and collision energy were optimized for each compound and the retention times were 2.13 and $1.44 \mathrm{~min}$ for IF and $\mathrm{OH}-\mathrm{CP}$, respectively. Compared to the corresponding control group (group 3 ), the amount of $\mathrm{OH}-\mathrm{CP}$ was about two times higher $(P<0.05)$ in tumors that had received 2 days of thalidomide treatment (Fig. 4a). In this figure, results are normalized in percentage, with $100 \%$ corresponding to the $\mathrm{OH}-\mathrm{CP}$ response in control tumors. The $\mathrm{OH}-$ $\mathrm{CP}$ response is the ratio between the $\mathrm{OH}-\mathrm{CP}$ and IF peaks response area. The values were $0.037 \pm 0.007$ and $0.072 \pm 0.012$ for groups 3 and 6 , respectively. This result confirms that the potentiation of chemotherapy was caused by an increase of the quantity of drug penetrating into the tumor. To discriminate between an effect on the tumor vasculature and a possible effect of increase in production of $\mathrm{OH}-\mathrm{CP}$ by the metabolism, we also performed a quantification of $\mathrm{OH}-\mathrm{CP}$ in circulating blood. Fig. 4b shows that the amount of this metabolite was not significantly different between the blood of treated and control mice $(P>0.05)$. In this figure, results are normalized in percentage, with $100 \%$ corresponding to the $\mathrm{OH}-\mathrm{CP}$ response in control tumors. The values were $0.207 \pm 0.023$ and $0.214 \pm$ 0.012 for groups 3 and 6 , respectively. Consequently, we can conclude that the increase in the amount of

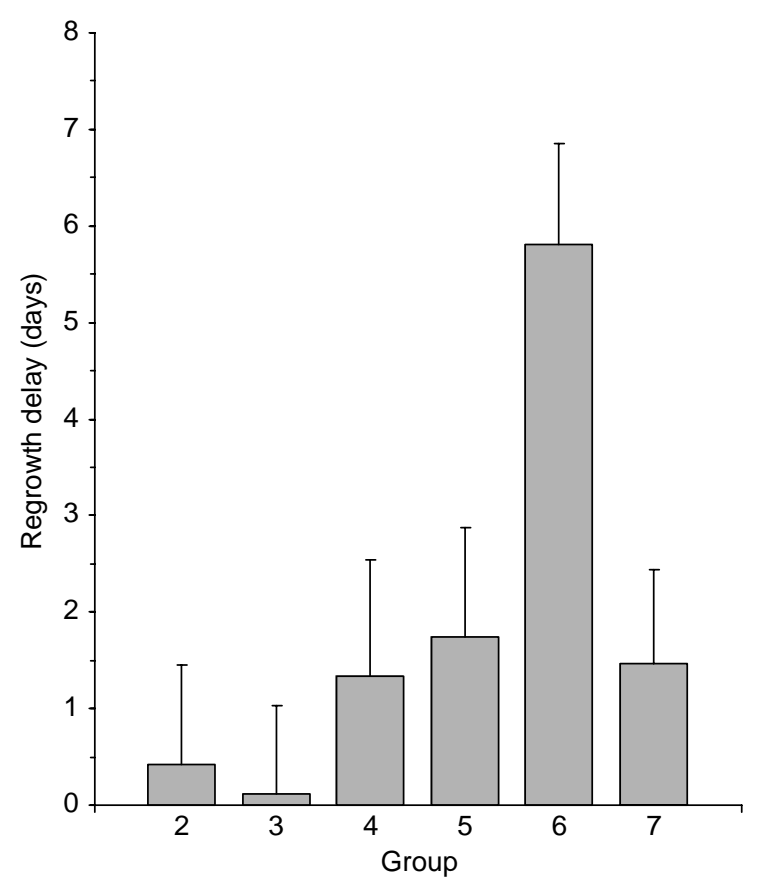

Fig. 2. Difference between re-growth delay of treated mice and mice treated with vehicle (DMSO). The re-growth delays observed using all modalities of treatments were not significant compared to the control group (group 1), except for the mice pre-treated with two daily injection of thalidomide prior to the administration of cyclophosphamide (group 6). 


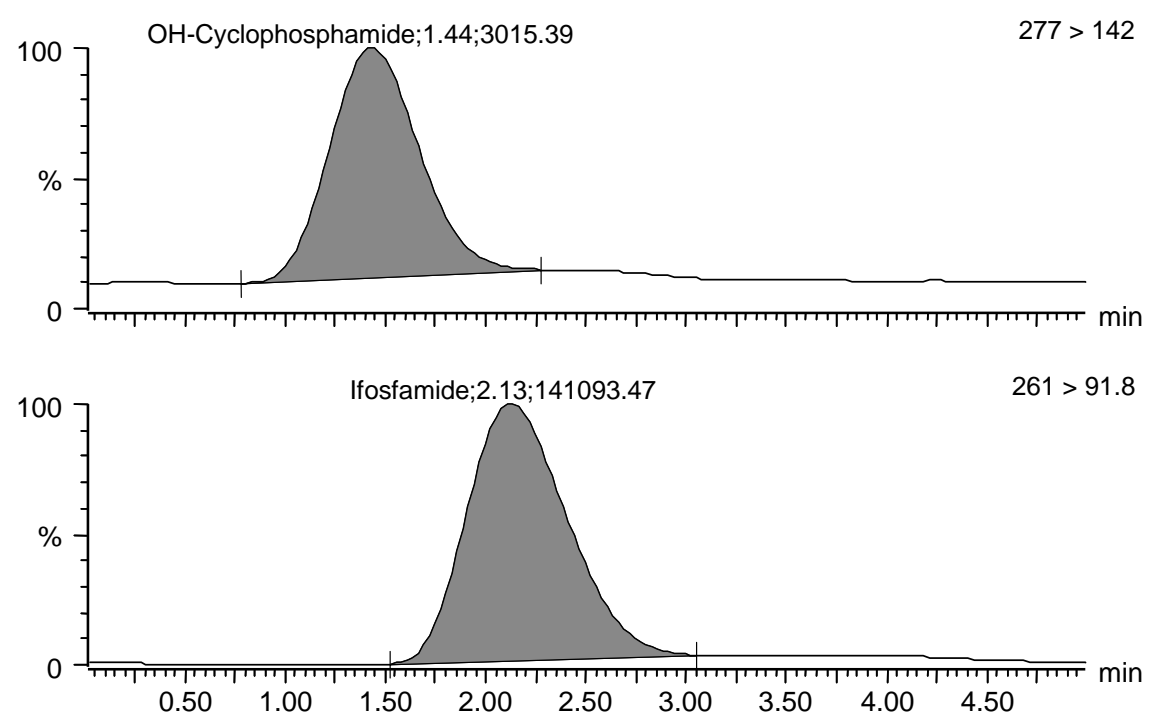

Fig. 3. HPLC/MS/MS chromatogram of a tumor sample. The values indicated near the compound name are the retention time and the area under the curve, respectively. On the right of each chromatogram is indicated the mutliple reaction mode (MRM) transition with the molecular mass of the ionized metabolite and the molecular mass of the fragmented daughter ion. The MRM transition and the retention time allow unambiguous identification of a molecule.

metabolite arriving in the tumor is not caused by an increase in the metabolism of cyclophosphamide, but by an increase in the delivery.

\section{Discussion}

The present study demonstrates that the benefit of combined therapy using an anti-angiogenic agent (for this study, thalidomide) with a cytotoxic agent (cyclophosphamide) requires knowledge of the time window during which the vessels initially become normalized. When the cytotoxic agent is administered outside this time window, no therapeutic benefit is obtained. This result is very important to consider for combination of anti-angiogenic therapies and chemotherapy. The benefit of such association has produced inconsistent findings: some studies have found an additive effect [16-18] while other have found a compromised effect $[19,20]$. This inconsistency may not be surprising, considering that these studies were designed without taking into account the recent evidence for the 'normalization' of the tumor vasculature at the early phase of an anti-angiogenic treatment $[6,7]$. For combined anti-angiogenic/radiation therapy, a clear benefit of a schedule that takes into account the 'normalization' window was demonstrated in glioblastoma using DC101 (VEGFR2-specific monoclonal antibody) [23], and in fibrosarcoma using thalidomide [8]. Anti-angiogenic treatments have also been found to increase the penetration of several molecules when administered during the 'normalization' window: serum albumin [22], Hoechst 33342 dye [21], Patent Blue dye [8], Gd contrast agent [8], and the cytotoxic agent CPT-11 [21]. However, so far no study has been carried out to correlate the kinetics of tumor vascular
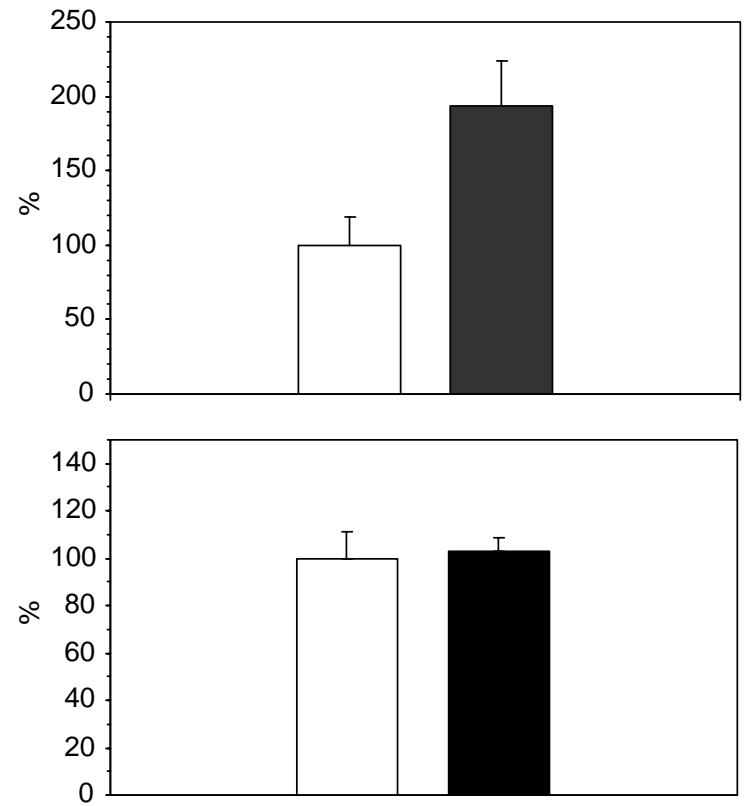

Fig. 4. Estimation of the amount of OH-CP by HPLC/MS/MS in tumors (a) and blood (b) of normalized tumors (dark) and control (open). The number of tumor bearing mice in each of the four groups was 10 . 
'normalization' with a therapeutic benefit when antiangiogenic treatment is combined with chemotherapy. Our present study was specifically designed to assess the importance of the schedule timing when combining thalidomide together with cyclophosphamide.

EPR oximetry was used to determine the timing of the 'normalization' window. The early increase in tumor oxygenation found in hepatocarcinoma (Fig. 1) was rather comparable to the timing observed in fibrosarcoma [8]. Having identified this time window, we tested several combinations of thalidomide and cyclophosphamide, which differed in the timing of administration: cytotoxic agent before, during or after thalidomide. Our results are remarkable in a sense that only the administration during the 'normalization' period led to a therapeutic benefit (Fig. 2).

To demonstrate that the therapeutic benefit is really due to an increase in the delivery of the cytotoxic agent, we used HPLC/MS/MS to determine the amount of active compound in tumors. Cyclophosphamide is a prodrug of a DNA alkylating agent. To be active, cyclophosphamide must be metabolized by the P450 system in the liver by addition of a hydroxy group to form the 4-hydroxyclophosphamide (OH-CP). In mice, $90 \%$ of the $\mathrm{CP}$ administered is activated to $\mathrm{OH}-\mathrm{CP}$, with a very rapid rate of activation $\left(k_{\mathrm{a}}=8.4 \mathrm{~h}^{-1}\right)$ [24]. $\mathrm{OH}-\mathrm{CP}$ is released in the blood stream and is in equilibrium with its ring-opened tautomer, aldophosphamide. Aldophosphamide undergoes $\beta$-elimination of the unstable acrolein, yielding phosphoramide mustard (PM). While both 4-OH-CP and PM are cytotoxic in vitro and in vivo, the former does not function as an alkylating agent in physiological $\mathrm{pH}$. Thus, PM is considered the ultimate alkylating metabolite of CP. PM cannot cross cell membranes, however, so only the intracellularly formed fraction is cytotoxic $[12,13,25,26]$. Because the cell membrane is permeable to OH-CP [25-27], the intracellular concentration of phosphoramide mustard is consequently directly proportional to the amount of $\mathrm{OH}-\mathrm{CP}$ delivered to the tumor. In this metabolic pathway, $\mathrm{OH}-\mathrm{CP}$ functions as a transport molecule that delivers the polar metabolite PM into the cells where alkylation takes place [28]. For all of these reasons, we chose to quantify $\mathrm{OH}-\mathrm{CP}$ as the marker of chemotherapy potentiation. The HPLC/MS/MS measurements showed that the quantity of $\mathrm{OH}-\mathrm{CP}$ penetrating into the tumor was greater in the mice treated by thalidomide than for controls (Fig. 4). Finally, it was necessary to determine the cause for the increased amount of $\mathrm{OH}-\mathrm{CP}$ in tumors, and to discriminate between an increase in delivery of $\mathrm{OH}-\mathrm{CP}$ (via increased perfusion) and an increase in production of $\mathrm{OH}-\mathrm{CP}$ by liver metabolism. Indeed, thalidomide has been shown to stimulate the production of P-450 in the rat liver during chronic treatments (60 days) [29]. We observed that the quantity of $\mathrm{OH}-\mathrm{CP}$ was not significantly changed in the blood of treated mice relative to controls (Fig. 4), a result that is consistent with the very short time of treatment. Consequently, we can state unambiguously that the higher therapeutic efficacy is due to an increase in the delivery of $\mathrm{OH}-\mathrm{CP}$ to the tumor, and not to a stimulation of the metabolism of cyclophosphamide.

An issue related to our study is whether tumor oxygenation merely reflects the increase in perfusion during the 'normalization' of the vasculature, or if oxygen also plays a role in the increase in sensitivity of tumors to cyclophosphamide. Several previous chemotherapy studies (although none as yet using cyclophosphamide) have shown that oxygen can indeed, albeit in an indirect manner, modulate the efficacy of chemotherapy. As a result of a decline of nutrient and oxygen availability, cells farther away from the vascular system may divide at a reduced rate and thus be protected from the effects of chemotherapeutic agents whose activity is selective for rapidly dividing cell populations. Consequently, the increase of oxygen availability could improve the cytotoxic effect of a chemotherapeutic drug. Therefore, we cannot exclude the hypothesis that the increase in oxygen caused by thalidomide plays an additive role in the potentiation of chemotherapy by cyclophosphamide.

In conclusion, the study of changes in the tumor microenvironment during anti-angiogenic treatments is of crucial importance for planning the combination of such drugs with chemotherapy. The optimization of schedule in patients will benefit from recent developments in spectroscopy/imaging technologies such as EPR [30], MRI [31], PET [32] or other surrogate markers such as measurements of interstitial fluid pressure [33] that will allow the determination of the 'normalization' window.

\section{Acknowledgements}

This work is supported by grants from the Belgian National Fund for Scientific Research (FNRS), the Télévie, the Fonds Joseph Maisin, and the 'Actions de Recherches Concertées-Communauté Française de Belgique-ARC 04/09-317'. OF is a Research associate of the FNRS. 


\section{References}

[1] A. Bikfalvi, Significance of angiogenesis in tumor progression and metastasis, Eur. J. Cancer 31 (1995) 1101-1104.

[2] P. Carmeliet, R.K. Jain, Angiogenesis in cancer and other diseases, Nature 407 (2000) 249-257.

[3] M.S. O'Reilly, L. Holmgren, C. Chen, J. Folkman, Angiostatin induces and sustains dormancy of human primary tumors in mice, Nat. Med. 2 (1996) 689-692.

[4] S.S. Yoon, H. Eto, C.M. Lin, H. Nakamura, T.M. Pawlik, S.U. Song, K.K. Tanabe, Mouse endostatin inhibits the formation of lung and liver metastasis, Cancer Res. 59 (1999) 6251-6256.

[5] J.C. Yang, L. Haworth, R.M. Sherry, P. Hwu, D.J. Schwartzentruber, S.L. Topalian, et al., A randomized trial of bevacizumab, an anti-vascular endothelial growth factor antibody, for metastatic renal cancer, N. Engl. J. Med. 349 (2003) 427-434.

[6] R.K. Jain, Normalization of tumor vasculature: an emerging concept in antiangiogenic therapy, Science 307 (2005) 58-62.

[7] R.K. Jain, Normalizing tumor vasculature with anti-angiogenic therapy: a new paradigm for combination therapy, Nat. Med. 7 (2001) 987-989.

[8] R. Ansiaux, C. Baudelet, B.F. Jordan, N. Beghein, P. Sonveaux, J. De Wever, et al., Thalidomide radiosensitizes tumors through early changes in the tumor microenvironment, Clin. Cancer Res. 11 (2005) 743-750.

[9] H.S. Taper, G.W. Woolley, M.N. Teller, M.P. Lardis, A new transplantable mouse liver tumor of spontaneous origin, Cancer Res. 26 (1966) 143-148.

[10] B. Gallez, B.F. Jordan, C. Baudelet, P.D. Misson, Pharmacological modifications of the partial pressure of oxygen in murine tumors: evaluation using in vivo EPR oximetry, Magn. Reson. Med. 42 (1999) 627-630.

[11] A. Paci, A. Rieutord, F. Brion, P. Prognon, Separation methods for alkylating antineoplastic compounds, J. Chromatogr. B 764 (2001) 255-287.

[12] N. Sadagopan, L. Cohen, B. Roberts, W. Collard, C. Omer, Liquid chromatography-tandem mass spectrometric quantitation of cyclophosphamide and its hydroxy metabolite in plasma and tissue for determination of tissue distribution, J. Chromatogr. B 759 (2001) 277-284.

[13] A.D.R. Huitema, M.M. Tibben, T. Kerbusch, J.J. Kettenes-van den Bosch, S. Rodenhuis, J.H. Beijen, Simple and selective determination of cyclophosphamide metabolite phosphoramide mustard in human plasma using high-performance liquid chromatography, J. Chromatogr. B 745 (2000) 345-355.

[14] C. Sottani, R. Turci, L. Perbellini, C. Minoia, Liquid-liquid extraction procedure for trace deremination of cyclophosphamide in human urine by high-performance liquid chromatography tandem mass spectrometry, Rapid Commun. Mass Spectrom. 12 (1998) 1063-1068.

[15] B. Gallez, C. Baudelet, B.F. Jordan, Assessment of tumor oxygenation by electron paramagnetic resonance: principles and applications, NMR Biomed. 17 (2004) 240-262.

[16] B.A. Teicher, S.A. Holden, G. Ara, N.P. Dupuis, F. Liu, J. Yuan, et al., Influence of an antiangiogenic treatment on 9L gliosarcoma: oxygenation and response to cytotoxic therapy, Int. J. Cancer 61 (2005) 732-737.
[17] E. Heere-Ress, J. Boehm, C. Thallinger, C. Hoeller, V. Wacheck, P. Birner, et al., Thalidomide enhances the antitumor activity of standard chemotherapy in a human melanoma xenotransplatation model, J. Invest. Dermatol. 125 (2005) 201-206.

[18] Q. Ding, P. Kestell, B.C. Baguley, B.D. Palmer, J.W. Paxton, G. Muller, L.-M. Ching, Potentiation of the antitumour effect of cyclophosphamide in mice by thalidomide, Cancer Chemother. Pharmacol. 50 (2002) 186-192.

[19] R. Murata, Y. Nishimura, M. Hiraoka, An antiangiogenic agent (TNP-470) inhibited reoxygenation during fractionated radiotherapy of murine mammary carcinoma, Int. J. Radiat. Oncol. Biol. Phys. 37 (1997) 1107-1113.

[20] J. Ma, S. Pulfer, S. Li, J. Chu, K. Reed, J.M. Gallo, Pharmacodynamic-mediated reduction of temolozomide tumor concentrations by the angiogenesis inhibitor TNP-470, Cancer Res. 61 (2001) 5491-5498.

[21] H. Wildiers, G. Guetens, G. De Boeck, E. Verbeken, B. Landuyt, W. Landuyt, et al., Effect of antivascular endothelial growth factor treatment on the intratumoral uptake of CPT-11, Br. J. Cancer 88 (2003) 1979-1986.

[22] R.T. Tong, Y. Boucher, S.V. Kozin, F. Winkler, D.J. Hicklin, R.K. Jain, Vascular normalization by vascular endothelial growth factor receptor 2 blockade induces a pressure gradient across the vasculature and improves drug penetration in tumors, Cancer Res. 64 (2004) 3731-3736.

[23] F. Winkler, S.V. Kozin, R.T. Tong, S.S. Chae, M.F. Booth, I. Garkavtsev, et al., Kinetics of vascular normalization by VEGFR2 blockade governs brain tumor response to radiation: role of oxygenation, angiopoietin-1, and matrix metalloproteinases, Cancer Cell 6 (2004) 553-563.

[24] M.J. Moore, Clinical pharmacokinetics of cyclophosphamide, Clin. Pharmacokinet. 20 (1991) 194-208.

[25] N. Brock, H.J. Hohorst, Metabolism of cyclophosphamide, Cancer 20 (1967) 900-904.

[26] B.E. Domeyer, N.E. Sladek, Kinetic of cyclophosphamide biotransformation in vivo, Cancer Res. 40 (1980) 174-180.

[27] F. Baumann, R. Preiss, Cyclophosphamide and related anticancer drugs, J. Chromatogr. B 764 (2001) 173-192.

[28] G. Voelcker, T. Wagner, C. Wientzek, H.J. Hohorst, Pharmacokinetics of 'activated' cyclophosphamide and therapeutic efficacies, Cancer 15 (1984) 1179-1186.

[29] D. Tsambaos, K. Bolsen, S. Georgiou, A. Monastirli, G. Goerz, Effects of oral thalidomide on rat liver and skin microsomal $\mathrm{P} 450$ isozyme activities and on urinary porphyrin excretion: interaction with oral hexachlorobenzene, Arch. Dermatol. Res. 286 (1994) 347-349.

[30] H.M. Swartz, N. Khan, J. Buckey, R. Comi, L. Gould, O. Grinberg, et al., Clinical applications of EPR: overview and perspectives, NMR Biomed. 17 (2004) 335-351.

[31] A.R. Padhani, MRI for assessing antivascular cancer treatments, Br. J. Radiol. 73 (2003) 60-80.

[32] J.G. Rajendran, K.A. Krohn, Imaging hypoxia and angiogenesis in tumors, Radiol. Clin. N. Am. 43 (2005) 169-187.

[33] M. Milosevic, A. Fyles, R. Hill, Interstitial fluid pressure in cervical cancer: guide to targeted therapy, Am. J. Clin. Oncol. 24 (2001) 516-521. 